\title{
Glomeluar Filtration Rate from Cystatin C and Creatinine Adjusted for BSA
}

National Cancer Institute

\section{Source}

National Cancer Institute. Glomeluar Filtration Rate from Cystatin C and Creatinine

Adjusted for BSA. NCI Thesaurus. Code C127614.

The determination of the glomerular filtration rate by measurement of creatinine and cystatin C clearance and whose result is adjusted for body surface area. 